Ma. Melizza S. Villalon, MD

Lei-Joan Vital, MD

Department of Otorhinolaryngology

Head and Neck Surgery

Veterans Memorial Medical Center
Correspondence: Dr. Lei-Joan Vital

Department of Otorhinolaryngology

Head and Neck Surgery

Veterans Memorial Medical Center

North Avenue, Diliman, Quezon City 0870

Philippines

Phone: (632) 4269775

Email:enthns_vmmc@yahoo.com

Reprints will not be available with the author

The authors declared that this represents original materia that is not being considered for publication or has not been published or accepted for publication elsewhere in full or in part, in print or electronic media; that the requirements for authorship have been met by each author, and that each author believes that the manuscript represents honest work.

Disclosures: The authors signed disclosures that there are no financial or other (including personal) relationships, intellectual passion, political or religious beliefs, and institutional affiliations that might lead to a conflict of interest.

\section{Compound Odontoma of the Maxillary Sinus}

In 1863, the term odontoma was introduced by Paul Broca which he described as a tumor formed by overgrowth of transitory or complete dental tissue. The World Health Organization classified them under mixed benign odontogenic tumors because of their origin from epithelial and mesenchymal cells exhibiting different structures of dental tissue (enamel, dentin, cementum and pulp). ${ }^{1}$ There are two distinct types: compound and complex. Compound odontoma is composed of all odontogenic tissue in an orderly fashion resulting in many teeth-like structures but with no morphological resemblance to normal teeth whereas a complex odontoma appears as an irregular mass with no similarity even to rudimentary teeth. ${ }^{2,3,4}$

The pathogenesis of odontomas has not been completely established although the most accepted etiology is related to trauma, infection, growth pressure and genetic mutations in one or more genes that cause disturbances in the mechanism controlling tooth development. ${ }^{1,5}$

Patients with compound odontoma are often asymptomatic. It is usually detected on routine radiography upon examination of an unerrupted tooth. ${ }^{6}$ Odontomas can occur anywhere in the jaws and are usually found associated with or within the alveolar process. ${ }^{7}$ However, the presence of an odontoma in the maxillary sinus is very rare.

We present a female patient with a compound odontoma in the maxillary sinus initially managed as nasal vestibulitis with maxillary sinusitis.

\section{CASE REPORT}

A 63-year-old woman from Cavite City, Philippines consulted in our institution due to perception of foul odor. Six weeks prior to admission, she experienced right alar pain, facial fullness and swelling with associated undocumented fever. She consulted an ENT specialist and was diagnosed with nasal vestibulitis with maxillary sinusitis. She was given cefixime $200 \mathrm{mg}$, one tablet twice a day and Metronidazole 500mg, one tablet every six hours for seven days.

Five weeks prior to admission, despite resolution of the nasal and maxillary swelling and pain, she started to perceive a foul odor. There was no associated nasal congestion and nasal discharge, fever, no nasal itchiness nor frequent sneezing. Her physician requested an orthopantomogram hat revealed a suspicious mass and haziness in the right maxillary sinus and an impacted tooth in the left maxillary sinus. (Figure 1) She was advised surgery but opted for a second opinion.

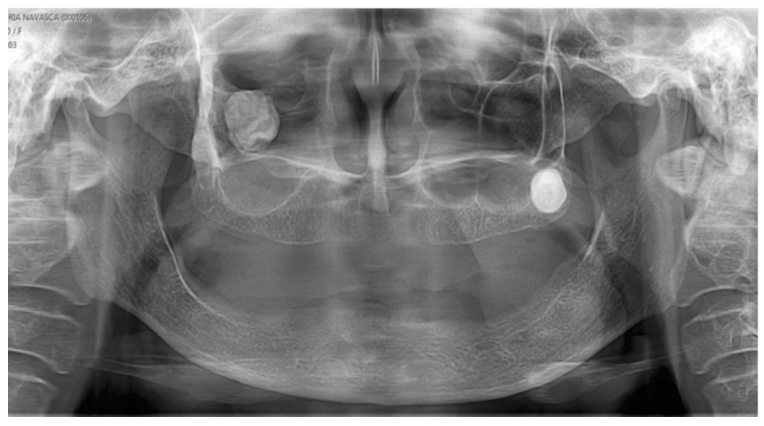

Figure 1. Orthopantomogram of the paranasal sinuses showing a suspicious mass and haziness in the right maxillary sinus and an impacted tooth in the left maxillary sinus. 
Two weeks prior to admission and still with perception of foul odor, she consulted another ENT specialist and was given co-amoxiclav $625 \mathrm{mg}$, one tablet every eight hours. A CT scan of the paranasal sinuses revealed mucoperiosteal thickening and calcific density within the opacified right maxillary sinus. (Figure $2 A, B$ ) The patient was advised surgery.

The patient had pulmonary tuberculosis in 1983 but was treated for six months. She does not recall having any un-erupted teeth and claimed that her previous dental extractions were unremarkable. She had a family history of bronchial asthma and colon cancer. She did not drink alcoholic beverages but she previously smoked for one packyear.

Anterior rhinoscopy revealed scant clear mucoid discharge in both nasal cavities, noncongested and nonhyperemic turbinates and no
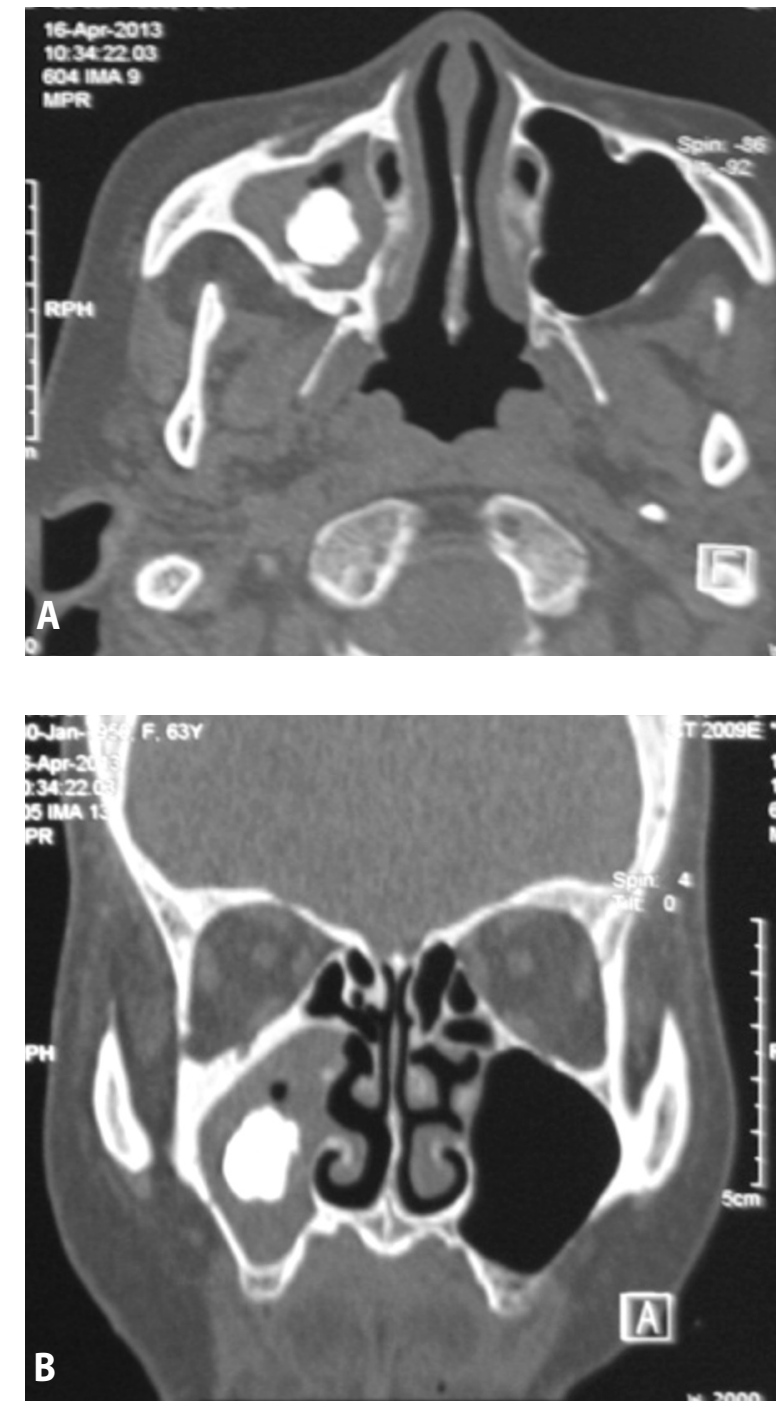

Figure 2. CT scan of the paranasal sinuses. A. axial view and B. coronal view showing mucoperiosteal thickening and calcific density within the opacified right maxillary sinus. intranasal mass. She was edentulous with no facial mass or swelling. The rest of the examination was unremarkable.

With an assessment of a right maxillary mass (odontogenic tumor versus foreign body) with right maxillary sinusitis and an impacted tooth in the left maxilla she underwent a Caldwell-Luc procedure. Antrotomy was performed through the canine fossa via a gingivolabial incision overlying the anterior maxillary wall. Thick clear mucous was seen oozing out and eventually drained and suctioned out. (Figure 3) A $2 \mathrm{~cm} \times 2 \mathrm{~cm} \times 2.1 \mathrm{~cm}$ ovoid, whitish to tan colored hard mass partially covered by black fragments was carefully extracted. (Figure 4) Irrigation of the maxillary sinus was performed using normal saline solution and the natural maxillary ostium was widened. The incision was closed with interrupted mattress sutures using chromic 3.0 and the mass was submitted for histopathological analysis.

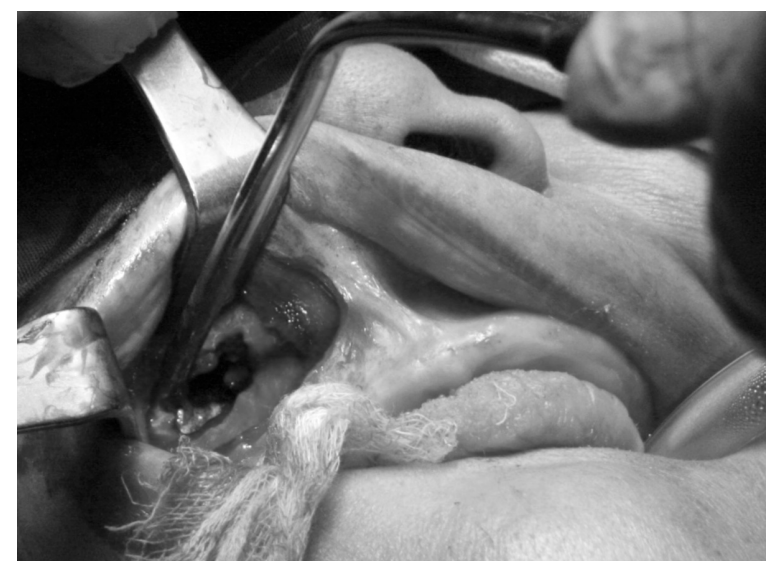

Figure 3. Caldwell-Luc procedure exposing the right maxillary antrum

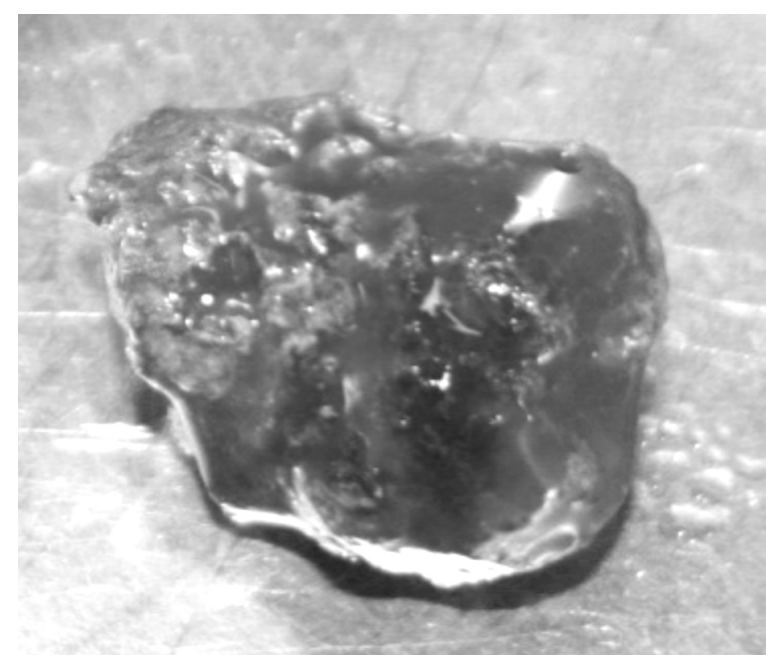

Figure 4. Macroscopic appearance of compound odondoma located in the maxillary sinus as seen in the patient-a $2 \times 2 \times 2.1 \mathrm{~cm}$ tan, white, ovoid hard mass partially covered by black fragments. 


\section{FEATURED GRAND ROUNDS}

Microscopic sections revealed misshapen teeth or denticles with a coordinated pattern of calcification such as enamel, dentin and cementum. (Figure 5A-C) The final histopathologic report was a compound odontoma of the right maxillary sinus.

The postoperative follow-up was satisfactory. Our patient developed no oro-antral fistula and showed no signs of maxillary sinusitis and the perception of foul odor resolved.
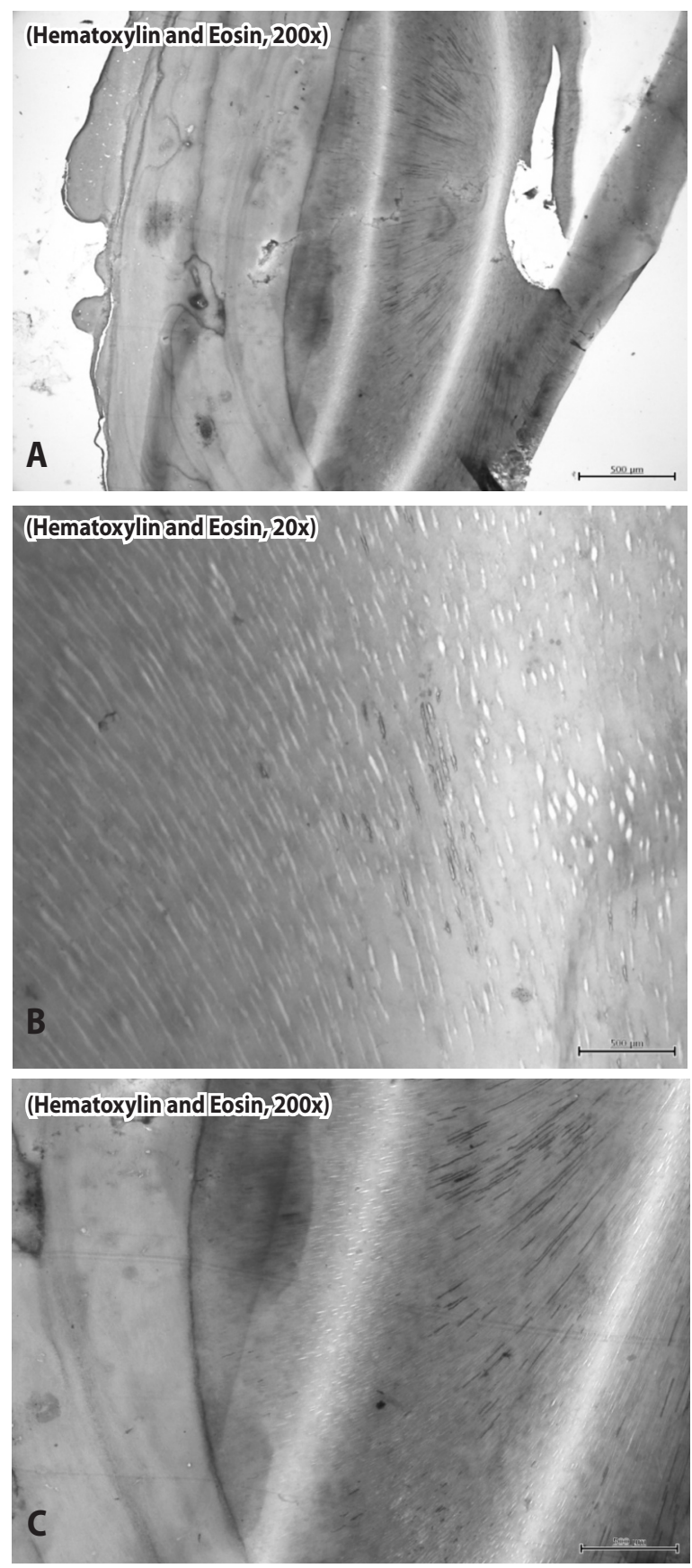

Figure 5. Microscopic appearance of compound odondoma located in the maxillary sinus. A. Enamel, dentin and cementum (H\&E; 200x) B. Denticles (H\&E); 20x C. Enamel, dentin and cementum (H\&E; 200x)

\section{DISCUSSION}

Odontoma is a generally asymptomatic, slowly progressing tumor that may pass unnoticed. It is usually detected by routine radiograph. This may be associated with un-erupted tooth mainly the mandibular third molar followed by the upper canine and upper central incisor. The prevalence of odontoma associated with impacted canine is $1.5 \%{ }^{8}$ The maxillary sinus is a frequent site for pathologies of odontogenic origin because of its close anatomical relationship with teeth and periodontal tissues. This makes a frequent but not a common site for inflammatory diseases as well as neoplastic lesions. ${ }^{6}$ The patient initially presented with right alar pain and right facial swelling. She did not recall having an un-erupted tooth and claimed that her previous dental extractions were unremarkable. After treatment, the pain and swelling resolved but she started to perceive a malodorous smell. Commonly, clinicians arrive at the diagnosis of sinusitis when failure of its resolution despite antibiotic treatment prompts warning bells that warrant further radiographic investigation. The radiographic appearance of odontoma is almost always diagnostic ${ }^{3}$ as in the presented case. Panoramic and periapical images usually show well-defined borders of a similar density to calcified dental tissue, having a ground-glass appearance, and a radiopaque mass occupying the affected maxillary sinus. ${ }^{9}$ This was evident in the patient's panoramic radiograph.

Additional radiographic evaluation with computed tomography was necessary to determine the extension and features of the lesion because periapical and panoramic images do not provide complete visualization of the maxillofacial complex. CT scans serve as a guide not only for evaluation of the lesion itself but also for localization of associated pathology and proper treatment planning. ${ }^{10}$ In this case, the computed tomography scan of the paranasal sinuses revealed mucoperiosteal thickening and calcific density within the opacified right maxillary sinus suggesting odontogenic origin with concomitant maxillary sinusitis.

Due to its asymptomatic course, it can be surmised that the patient might have had the asymptomatic compound odontoma for a long time. The mass in her maxillary sinus was seen freely floating in her CT scan. It may be hypothesized that obstruction by the odontoma could have altered the ventilation and drainage of the maxillary sinus causing the symptoms of the patient. Cabov, et al. reported that odontomas in the maxillary sinus may also cause pain, facial asymmetry and chronic congestion of the sinus. ${ }^{11}$

Management for this case was surgical removal of the mass with drainage of trapped mucus as well as medical treatment of the maxillary sinus infection. The Caldwell-Luc procedure was the favored approach to this case because it offered easy access to the mass that could not be extracted trans-nasally because of its size and solid nature. Restoring 


\section{FEATURED GRAND ROUNDS}

the drainage of the maxillary sinus was also essential and this was done by widening the natural maxillary sinus ostium.

The histological characteristics of the mass extracted from the patient consisted of denticles with a coordinated pattern of calcification such as enamel, dentin and cementum compatible with a compound odontoma.

The rarity of odontomas makes them easy to miss should a radiographic examination not have been done. Despite their being usually asymptomatic, our patient had chronic perception of foul odor that was bothersome and frustrating. A clinician relying on medical history and physical examination alone could not have arrived at the correct diagnosis. In this case, it was shown that radiographic imaging was very crucial in catching a hidden and rare tumor.

1. Ajike SO, Adekeye EO. Multiple odontomas in the facial bones. A case report. Int J Oral Maxillofac Surg. 2000 Dec: 29(6):443-4.

2. Hanemann JA, Oliveira DT, Garcia NG, Santos MR, Pereira AA. Peripheral compound odontoma erupting in the gingiva. Head Face Med. 2013 Jun 11; 9:15.

3. Prabhakar C, Haldavnekar S, Hegde S. Compound- Complex odontoma - An important clinical entity. J Int Oral Health. 2012; 4(1)49-53.

4. Kramer IRH, Pindborg JJ, Shear M. Histological typing of odontogenic tumours. 2nd ed. Berlin Springer-Verlag. 1992: 16-21.

5. Hidalgo-Sánchez O, Leco-Berrocal MI, Martinez-González JM. Metaanalysis of the epidemiology and cinical manifestations of odontomas. Med Oral P Patol Oral Cir Bucal. 2008 Nov 1:13(11), 730-4.

6. Tan TY, Shashinder S, Subrayan V, Krishnan G. Silent sinus syndrome due to a maxillary mucocele. Auris Nasus Larynx. 2008 Jun; 35(2): 285-287.

7. Caton RB, Marble HB Jr, Topazian RG. Complex odontoma in the maxillary sinus. Oral Surg Oral Med Oral Pathol. 1973 Nov; 36(5): 658-662.

8. Malhotra K, Namdev R, Rohilla M, Dutta S. Unerupted Maxillary Central Incisor Associated with Compound Composite Odontoma: A Case Report. J Oral Health Comm Dent. 2012; 6(1) 43-46.

9. Au-Yeung KM, Ahuja AT, Ching AS, Metreweli C. Dentascan in oral imaging. Clin Radiol. 2001 Sep; 56(9): 700-713.

10. Dagistan S, Cakur B, Goregen M. A dentigerous cyst containing an ectopic canine tooth below the floor of the maxillary sinus: a case report. J Oral Sci. 2007 Sep; 49(3): 249-252.

11. Cabov T, Krmpotic M, Grgurevic J, Peric B, Jokic D, Spomenka Manojlovic S . Large complex odontoma of the left maxillary sinus. Wien Klin Wochenschr. 2005 Nov; 117(21-22):780-3. 